\title{
Epidémiologie de la Babésiose (Piroplasmose) canine dans le Sud-Ouest de la France
}

\author{
par J. BAILENGER et I. JAMIN
}

[Laboratoire de Zoologie et Biologie animale ( $\mathrm{P}^{\mathrm{r}}$ J. BAILENGER)

de la Faculté de Médecine et de Pharmacie, F 33 -Bordeaux]

\section{Résumé}

La Babésiose canine se manifeste avec une fréquence élevée dans tout le Sud-Ouest de la France, que la Garonne divise schématiquement en deux zones épidémiologiquement distinctes: au Nord la parasitose est intense et automno-hivernale; le Sud est moins atteint, mais avec une prédominance estivo-printanière.

Dermacentor reticulatus semble être le principal vecteur.

\section{Summary}

Canine babesiosis happens with a particularly high frequency in all the South West of France which the Garonne schematically divide in two epidemiologically definite regions. In the North, the disease is important and automno-hibernal. In the South, rate of infestation is much lower, but prevails during Spring and Summer.

Dermacentor reticulatus appears to be the prevalent vector of this infestation. 
La babésiose est une maladie cosmopolite dont l'importance justifie la part qu'elle prit dans les travaux du premier Congrès international de Parasitologie qui s'est tenu à Rome en 1964. L'absence de communication française, dans la section consacrée à ce sujet, pourrait amener à penser que cette parasitose est négligeable en France.

Au cours d'une étude publiée en 1962, Condoret, Bailenger et Amyot (2) ont attiré l'attention sur la fréquence de la babésiose canine dans la région de Bordeaux et ont analysé ses conditions épidémiologiques. Le présent travail étend à huit départements la mise au point sur l'état de cette parasitose dans le Sud-Ouest de la France et comporte une exploration particulière en Dordogne.

Une enquête a été faite auprès de 383 vétérinaires pratiquant dans les Basses-Pyrénées, le Gers, les Landes, le Lot-et-Garonne, la Gironde, la Dordogne, la Charente et la Charente-Maritime. 270 d'entre eux, soit $70 \%$, ont répondu en fournissant les précisions suivantes :

- Nombre de cas annuels;

- Mois de fréquence maximum ;

- Espèces canines les plus atteintes ;

- Caractéristiques de la région.

Ainsi, nous avons pu localiser les foyers, préciser l'intensité de leur atteinte et essayer de dégager certains facteurs épidémiologiques.

\section{Répartition et fréquence}

Près de 10.500 cas de babésiose canine nous ont été signalés en une seule année, de novembre 1962 à novembre 1963, par $70 \%$ des vétérinaires en exercice.

La répartition par département fait l'objet du tableau I. Il montre, avec la carte I, qui en est l'illustration plus précise, que la parasitose est générale, mais d'intensité variable : faible ou moyenne au Sud de la Garonne, elle est très élevée à partir de la Garonne et au Nord de celle-ci avec un maximum dans les territoires bordant les fleuves, rivières et leurs affiuents (Garonne, Dordogne, Isle, Charente...), ainsi que dans les régions marécageuses (Double, Marennes, Royan, Pons, Macau...).

L'existence de microzones très localisées, semblant indemnes de Babésiose canine, mérite de retenir tout particulièrement l'attention. On en trouve notamment en Chalosse (Sud du département des Landes), et dans la région des collines basques qui la prolonge au Sud, ainsi que dans le Confolentais (région Nord-Est de la Charente) et le Nontronnais (région Nord-Est de la Dordogne) qui, toutes deux, s'apparentent géographiquement au Limousin.

\section{Variations saisonnières}

Le caractère saisonnier de la babésiose canine est bien connu. Le Sud-Ouest n'échappe pas à la règle. Il est incontestable que l'intensité de la parasitose n'y est pas uniforme tout au long de l'année, mais il est très difficile, sinon impossible, d'établir une 
systématisation rigoureuse de la fréquence saisonnière d'autant que les variations annuelles sont sensibles. Sous ces réserves, on peut considérer qu'au Nord de la Garonne, c'est-à-dire dans la région la plus infestée, l'épidémie se développe le plus généralement de la mi-septembre à la mi-février et s'atténue ou même s'éteint, selon les

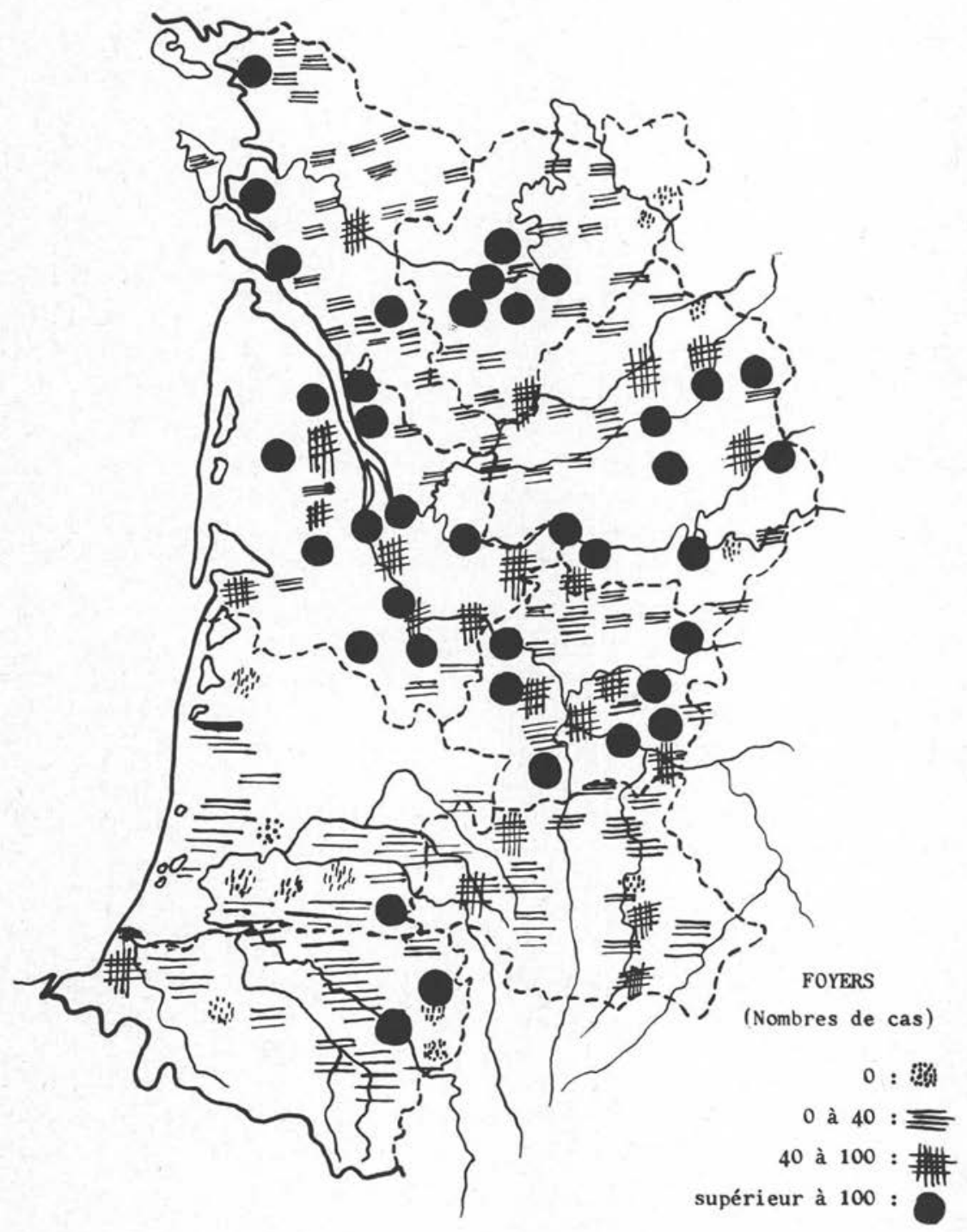

CARTe I. - La babésiose canine dans le Sud-Ouest de la France. Répartition et fréquence Annales de Parasitologie humaine et comparée (Paris), t. 43, 1968, $\mathrm{n}^{\circ} 1$ 


\section{TABleau I}

Nombre des cas de Babésiose canine de Novembre 1962 à Novembre 1963 communiqués par 270 vétérinaires

\begin{tabular}{|c|c|c|c|c|}
\hline DÉPARTEMENTS & (1) & (2) & NOMBre dE CAS & (3) \\
\hline Dordogne...$\ldots \ldots \ldots$ & 36 & 83 & 2.090 & 58 \\
\hline Gironde $\ldots \ldots \ldots \ldots$ & 43 & 71 & 2.500 & 58 \\
\hline Lot-et-Garonne...$\ldots$. & 33 & 70 & 1.865 & 56 \\
\hline Charente $\ldots \ldots \ldots \ldots$. & 32 & 69 & 1.240 & 39 \\
\hline Charente-Maritime... . & 40 & 66 & 1.270 & 32 \\
\hline Gers $\ldots \ldots \ldots \ldots \ldots$ & 31 & 68 & 600 & 20 \\
\hline Landes $\ldots \ldots \ldots \ldots$. & 25 & 69 & 390 & 15 \\
\hline Basses-Pyrénées ...... & 30 & 65 & 420 & 14 \\
\hline
\end{tabular}

(1) Nombre de vétérinaires ayant participé à l'enquête.

(2) Pourcentage des vétérinaires ayant participé à l'enquête par rapport aux vétérinaires en exercice dans le département considéré.

(3) Nombre moyen de cas de Babésiose canine par vétérinaire.

années, de mai à août ; tandis qu'au Sud du fleuve, allant de pair avec sa raréfaction, le parasitisme se manifeste plutôt au printemps et en été.

Le territoire que nous avons prospecté semble donc être divisé en deux zones schématiquement séparées par le cours de la Garonne :

- au Nord, la Babésiose canine est fréquente et automnale ;

— au Sud, elle est moins intense et estivo-printanière.

Cette opposition appelle une explication que l'on doit rechercher dans les conditions épidémiologiques. Malgré les multiples inconnues que comporte notre connaissance du cycle de Babesia (Piroplasma) canis, le rôle joué par les Ixodidés est indiscuté. L'épidémiologie de cette protozoose est ainsi dominée par la biologie des tiques vectrices. Il convient donc de déterminer les espèces d'Ixodidés en cause et de préciser les conditions géographiques caractéristiques des régions prospectées.

\section{Ixodidés}

Cette étude ne comporte que la détermination des Ixodidés en Dordogne. Elle complète celle que l'un de nous a précédemment effectuée en Gironde et qui portait sur 216 exemplaires prélevés sur des chiens malades (2). Une recherche similaire au Sud de la Garonne fera l'objet d'une publication ultérieure.

Une étroite collaboration établie avec les vétérinaires exerçant en Dordogne * nous a permis de recueillir, de novembre 1963 à novembre 1966, les tiques prélevées sur des chiens atteints de piroplasmose.

* Nous remercions tout particulièrement MM. les Drs Andrieux et Bugeaud (Excideuil), Dadoun (Miallet) Darpeix (Bergerac), Laplanche (Terrasson), Reveillas (Périgueux) et Sudrié (Thiviers). 
566 tiques ont ainsi pu être identifiées selon les critères retenus par Arthur (1) et Lamontellerie (3). Elles se répartissent entre les espèces suivantes :

Dermacentor reticulatus (Fabricius, 1794) .......... 470

Dermacentor marginatus, Sulzer, $1776 \ldots \ldots \ldots \ldots \ldots \ldots .38$

Rhipicephalus sanguineus, Latreille, $1806 \ldots \ldots \ldots \ldots \ldots . \ldots \ldots$

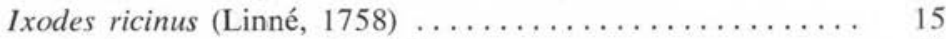

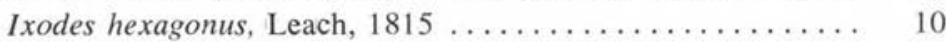

Dermacentor reticulatus apparaît donc comme l'espèce la plus souvent rencontrée. C'est une tique sauvage que nous avons trouvée de septembre à mai avec un maximum de fréquence d'ociobre à février. Sa répartition mensuelle est superposable à l'évolution saisonnière de la piroplasmose.

Dermacentor marginatus a de même été isolée en automne et en hiver et plus particulièrement en janvier-février.

Rhipicephalus sanguineus n'a été identifiée que dans le Sud du département (Bergerac et Montpon) et cela en automne ainsi qu'au début du printemps. A l'opposé des deux espèces précédentes, elle est absente pendant la période froide qui se situe en janvier-février.

L'activité saisonnière d'Ixodes ricinus est superposable à celle de $R$. sanguineus. Nous n'avons jamais trouvé cette tique seule sur les chiens malades, mais toujours associée à d'autres espèces : le plus souvent à $R$. sanguineus, plus rarement à $D$. reticulatus et exceptionnellement à $D$. marginatus.

L'importance de D. reticulatus avait déjà retenu notre attention lors de la prospection précédemment effectuée en Gironde. Cette espèce comptait 155 représentants sur les 216 tiques examinées. Nous l'avions alors dénommée $D$. marginatus, nous référant au travail de Lamontellerie (1954) qui les mettait en synonymie. La nomenclature actuelle en fait deux espèces distinctes. D'après Arthur (1), la différence repose notamment sur un éperon postéro-dorsal porté par le $2^{*}$ artic!e des palpes qui encadrent l'hypostome : il est développé chez $D$. reticulatus, réduit ou absent chez D. marginatus.

C'est à $D$. reticulatus qu'est le plus généralement dévolu le rôle primordial dans la transmission de la babésiose canine en France. L'intervention de Rhipicephalus sanguineus y est envisagée sans avoir été vérifiée.

Bien que nos déterminations aient été limitées à la Dordogne et à la Gironde, nous savons, par les travaux de Lamontellerie (3), que $D$. reticulatus a été trouvé dans sept des huit départements du Sud-Ouest soumis à notre prospection. Les Basses-Pyrénées ne figurent pas dans la carte de la répartition géographique de cette espèce. Quant aux localités landaises de référence, elles se situent essentiellement dans la portion du département occupée par la forêt. Dans la Chalosse qui s'apparente géographiquement et économiquement aux Basses-Pyrénées, seuls sont mentionnés : Dax qui occupe une position de transition aux confins des Landes et de la Chalosse, et Samadet au Sud-Est du département.

Ainsi, D. reticulatus considéré comme le vecteur essentiel de la babésiose canine 
est présent dans les régions du Sud-Ouest où cette parasitose sévit intensément avec une prédominance en automne-hiver. Il n'a pas encore été trouvé ni à l'Ouest de la Chalosse, ni dans les Basses-Pyrénées, régions où la babésiose canine est moins fréquente et prédomine au printemps et en été. Cette absence de $D$. reticulatus, ou tout au moins sa rareté, mérite des recherches poussées en raison de son importance épidémiologique.

\section{Facteurs épidémiologiques}

Leur analyse va nous conduire à discuter les explications qu'il est possible de fournir à la différence des intensités avec lesquelles la parasitose sévit dans les régions au Nord et au Sud de la Garonne.

Les variations d'intensité qui marquent la répartition de la babésiose canine dans le Sud-Ouest de la France semblent, en toute logique, devoir être essentiellement rapportées à l'activité des tiques responsables de la transmission de la maladie : la parasitose s'intensifie dans les zones offrant des conditions optimales pour leur développement. Leur sensibilité aux caractéristiques du milieu ambiant fait rechercher ces conditions parmi les facteurs climatiques et la végétation.

\section{$1^{\circ}$ Facteurs climatiques.}

Comme facteurs climatiques, nous avons retenu la pluviométrie, l'hygrométrie maximale et minimale, l'insolation, les températures (maximales, minimales et moyennes). Toutes ces données nous ont été fournies par la Météorologie nationale (station climatologique de Mérignac *). Elles correspondent à des moyennes établies sur une période de trente années (1921-1950), sauf en ce qui concerne l'insolation (1926-1935).

Seule, la carte des isohyètes présente une certaine analogie avec celle de la babésiose : la plus grande fréquence de cette parasitose coïncide avec les isohyètes inférieurs à 1.000 (voir carte II).

\section{$2^{\circ}$ Végétation.}

Les statistiques agricoles mises à notre disposition par le Ministère de l'Agriculture (Direction régionale de l'Institut national de la statistique) nous donnent l'aspect général des départements prospectés :

- La culture et les prairies dominent dans les Charentes, le Lot-et-Garonne et le Gers où elles représentent, par rapport à la superficie de chacun des départements, les pourcentages de 64,74 (Charente), 63,02 (Charente-Maritime), 59,76 (Lot-et-Garonne) et 71,53 (Gers).

- La Dordogne est un pays de bois (32,52 \% de la superficie) ; les châtaigniers t les chênes représentent le tiers des essences. Les terres incultes occupent une vaste surface $(14 \%)$; le reste est couvert de prairies ou mis en cultures.

\footnotetext{
* Nous remercions vivement M. Baudry, ingénieur de la météorologie, chef du bureau climatologique régional, des renseignements qu'il nous a aimablement communiqués.
} 


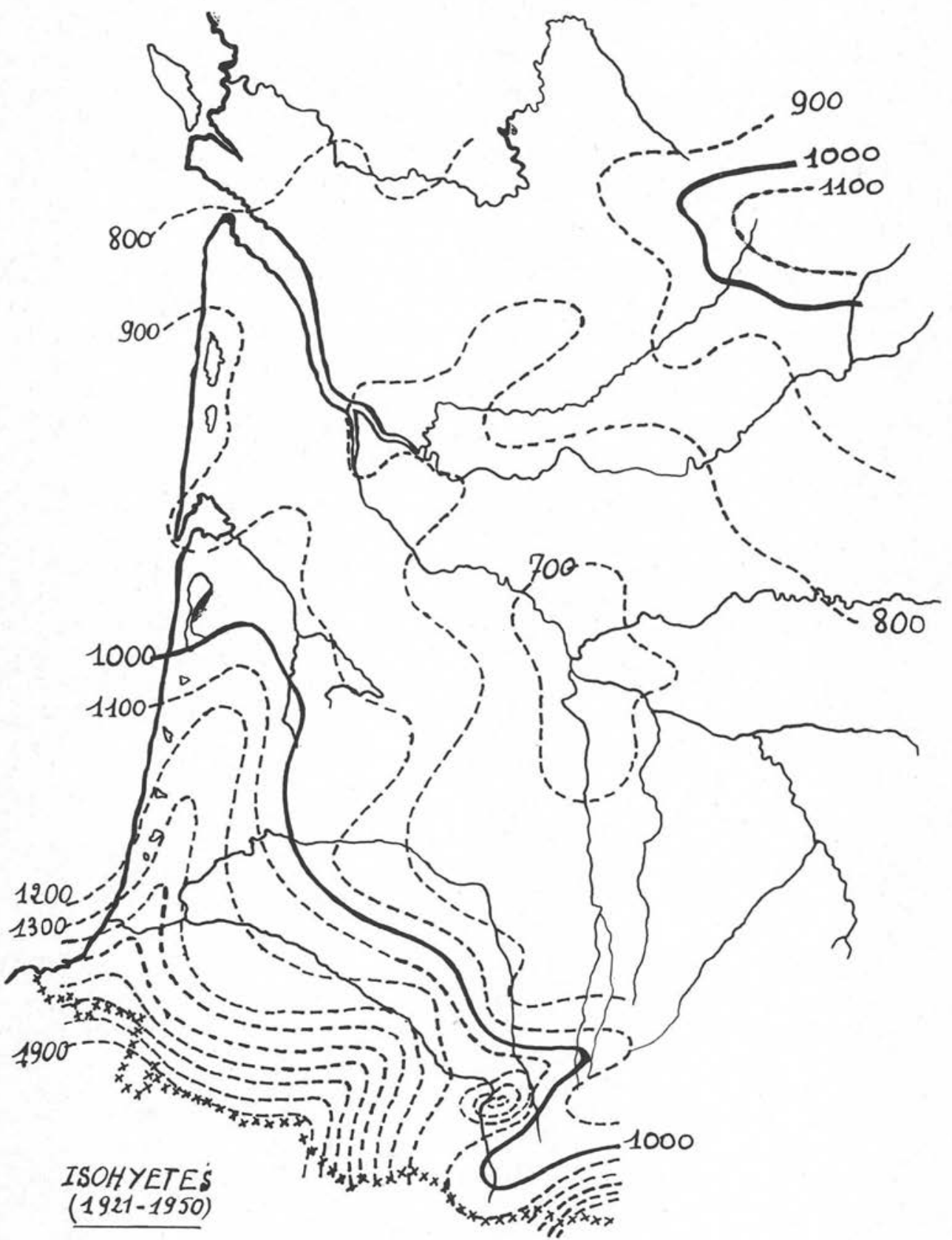

CARTE II. - Carte des Isohyètes (1921-1950). [Communiquée par la Météorologie Nationale (Station climatique de Mérignac)]. 
Ces statistiques sont d'interprétation délicate car elles ne révèlent pas d'oppositions géographiques tranchées, si ce n'est en ce qui concerne la pluviométrie. Cependant, il faut bien se garder de conclure, car elles reflètent une situation d'ensemble. Or, la taille des Ixodidés leur permet de se contenter de micro-climats résultant de la conjonction de l'humidité, de la température, de la végétation et de la nature du sol. Pour s'en convaincre, il suffit d'observer la carte de répartition de la babésiose canine que nous avons tracée: les zones marécageuses longeant les cours d'eau et ruisseaux naturels ou créés, sont les plus atteintes.

Nous sommes ainsi conduits à poser une hypothèse que nous allons nous attacher à vérifier : la piroplasmose sévit intensément lorsque les conditions d'évolution de Dermacentor reticulatus sont réalisées. La recherche de cette espèce dans les Pyrénées atlantiques (Chalosse + Basses-Pyrénées) doit nous permettre de confirmer ce point de vue.

Cependant, la présence de Dermacentor reticulatus ne semble pas suffisante. C'est ainsi que dans la région de Miallet (Nord-Est de la Dordogne), les chiens sont indemnes de babésiose, d'après le Docteur Dadoun. Ce vétérinaire affirme n'avoir jamais constaté cette parasitose, depuis plus de 10 ans qu'il est installé dans cette localité alors qu'il en avait soigné de nombreux cas lorsqu'il exerçait dans le Sud-Ouest de ce même département. Et pourtant, Dermacentor reticulatus y est abondant. Il manifeste d'ailleurs une agressivité particulière à l'égard de l'homme. La cause de ce comportement particulier de $D$. reticulatus tant à l'égard de Babesia canis qu'à l'égard de l'homme réside peutêtre dans la géologie qui isole cette région du Périgord calcaire et la rattache au Limousin en lui conférant un sol très acide, ainsi que dans le climat (Isohyète supérieur à 1.000 ) et dans la végétation qui est la conséquence des deux facteurs précédents.

\section{Conclusions}

Une enquête épidémiologique sur la babésiose canine effectuée dans huit départements du Sud-Ouest de la France, avec la collaboration de 270 vétérinaires, conduit à un certain nombre de notions qui ont pu être établies par l'étude de 10.500 cas relevés en une seule année.

La parasitose est générale, mais d'intensité variable et à recrudescence saisonnière. Ces deux caractères opposent deux zones schématiquement séparées par le cours de la Garonne: au Nord, la babésiose canine est fréquente et automno-hivernale ; au Sud, elle est moins intense et estivo-printanière.

Les Ixodidés prélevés sur des chiens malades appartiennent essentiellement à l'espèce Dermacentor reticulatus (155 sur 216 exemplaires en Gironde, 470 sur 566 exemplaires en Dordogne). L'activité de cette tique, généralement considérée comme le principal vecteur du parasite, se superpose à la recrudescence automno-hivernale de la maladie, dont les particularités épidémiologiques doivent être recherchées dans les exigences biologiques de cet acarien. 
Les statistiques établies par les services de la Météorologie nationale et une vue d'ensemble agricole des territoires ne rendent pas compte de la notion de micro-climat mieux adaptée à la biologie des Ixodidés ainsi qu'en témoigne la répartition de la babésiose canine qui suit le tracé des cours d'eau et se superpose à l'emplacement des zones marécageuses.

D. reticulatus est signalé par Lamontellerie dans toute la région que nous avons prospectée, à l'exception des Basses-Pyrénées. L'absence de cette espèce, ou du moins sa rareté dans cette zone, mérite confirmation car, si ce fait se révélait exact, les particularités épidémiologiques que nous avons individualisées (variations de fréquence du Nord au Sud et d'intensité saisonnière) trouveraient une explication.

\section{Bibliographie}

1. ARthur (R.), 1962. - Ticks and Disease, 445 p., Pergamon Press, London.

2. Condoret (A.), Bailenger (J.) et Amyot (B.), 1962. - La Piroplasmose dans le SudOuest de la France. Ann. Paras. hum. et comp., 37, 483-494.

3. Lamontellerie (M.), 1965. - Les tiques du Sud-Ouest de la France. Ann. Paras. hum. et comp., 40, 87, 100. 